\title{
Sexe, genre, éthique
}

\section{Karine Lambert}

Qu'est ce que le genre? Que désigne ce terme? On parle de genre grammatical. Le genre d'un nom signifie clairement que le masculin ou le féminin (sans parler du neutre) ne renvoie pas à une «nature » des choses. La chaise n'est pas plus féminine que le fauteuil n'est masculin, et si, en français, le soleil est masculin (au contraire de la lune, féminine), c'est l'inverse en allemand. Bref, le genre est une convention sociale. Le genre, terme commun à la grammaire, souligne le caractère construit et mobile dans le temps et l'espace de ce qui s'appelle ailleurs le sexe. Ce concept est utile pour penser la formation des catégories sociales. Ce mot connaît aujourd'hui des usages si fréquents qu'il est devenu chez les jeunes un tic de langage pour ponctuer une phrase. II s'est répandu dans toutes les sphères, y compris bien sûr celle de la science.

En matière de santé, femmes et hommes ne sont pas logés à la même enseigne. Mais les différences biologiques sont loin d'être seules en cause : les représentations sociales du féminin et du masculin influencent les patients, le corps médical et les chercheur.es. Depuis une vingtaine d'années, la recherche médicale internationale a intégré la question du genre dans ses pratiques et ses thématiques. De quelle façon les rôles sociaux et le contexte culturel influencent-ils la santé des femmes (et des hommes) ? Cette question répond à une exigence à la fois scientifique et éthique. II s'agit de mieux connaître les inégalités et les discriminations qui touchent les hommes et les femmes dans les domaines de la santé pour mieux les combattre. Or, pour appréhender la question du genre en santé, il faut d'abord définir ce concept car, en dépit de son succès sur différentes scènes sociales, le genre demeure souvent mal compris [16].

Le concept de genre a été introduit en France à la fin des années 1980. II sert à penser la construction sociale des sexes, des identités masculines et féminines. II aide à réfléchir dans toutes les disciplines, des sciences humaines et sociales aux sciences expérimentales et à comprendre comment les savoirs se sont construits.

Vignette (Photo @ Inserm - Frédérique Koulikoff et Alexandra Pinci).

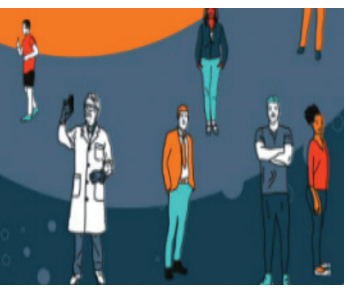

CNRS UMR 7303 TELEMMe (Temps, Espaces, Langages, Europe Méridionale - Méditerranée), équipe GeFem, université AixMarseille, 13000 Marseille, France.

klambert@univ-cotedazur.fr

\section{Que désigne le concept de genre?}

La réponse est inséparablement théorique et historique. Le genre est une catégorie qui déploie ses significations au cours d'une Histoire, et l'apport du concept de genre ne peut être appréhendé qu'à travers l'étude de ses usages. La circulation du genre d'un champ disciplinaire à l'autre amène à s'interroger sur la nature ambiguë du concept. Le genre n'est pas porteur d'une signification univoque, il est redéfini en fonction des contextes. Le concept est à la fois critique et normatif, il définit ce que sont les relations des sexes et ce qu'elles pourraient et devraient être. La différenciation des sexes fut longtemps à la fois présumée et ignorée par les sciences.

- Présumée comme une donnée première apparemment irréductible, rendant compte de la répartition sexuelle des rôles sociaux : en somme, aux hommes le public, aux femmes le privé.

- Ignorée dans la mesure où les expériences féminines étaient en quelque sorte supposées analogues à celles des hommes, et non susceptibles de réclamer des analyses particulières.

Illustrons-le par les recherches utilisant des animaux. Beaucoup d'expérimentations ont été conduites uniquement chez des mâles ${ }^{1,2}$. La raison invoquée était que les cycles hormonaux des femelles sont une source de variabilité des comportements. Ce n'est pas forcément vrai : des études montrent en effet que le principal facteur de variabilité dans les comportements des rongeurs est plutôt le nombre d'animaux confinés dans les cages, et non pas leur sexe.

La plupart des essais cliniques humains ont été menés chez des sujets masculins. On a même vu des recherches sur le risque de cancers gyné-

${ }^{1}$ Il y a quelques années, les autorités américaines se sont aperçues que $80 \%$ des médicaments retirés du marché l'avaient été en raison d'effets secondaires sur les femmes. Cela n'a rien de surprenant au demeurant, car les différentes phases d'essais portent en très grande majorité sur des sujets mâles.

https://www.sciencedirect.com/science/article/pii/S0149763410001156 https://lejournal.cnrs.fr/billets/la-sante-nest-pas-etrangere-au-genre

http://bigbrowser.blog.lemonde.fr/2014/05/15/science-faut-il-imposer-la-parite-chez-les-rats-delaboratoire/

${ }^{2}$ NDLR : Toutefois, en immunologie, qui est un champ majeur des sciences biologiques, on n'utilise très largement que des femelles, l'un des principaux arguments étant que les mâles s'affrontent et se blessent dans les cages. 
cologiques conduites chez des hommes! Face à de telles dérives, la législation rend désormais obligatoire de réaliser les essais cliniques sur des sujets des deux sexes, depuis 30 ans aux États-Unis et depuis 15 ans en Europe [1].

Pour autant «la femme », «les femmes » sont-elles en soi un objet de recherche? Non sans doute. On ne saurait étudier les femmes (ou les hommes) en général, mais des objets plus spécifiques : le corps, le travail, les rôles, les représentations et images des femmes... D'autre part, un sexe n'existe jamais que par rapport à l'autre et dans son rapport avec l'autre. Les recherches sur les femmes ou les hommes portent avant tout sur leurs rapports, individuels et collectifs, réels et symboliques. L'analyse de ces derniers dans les discours, les représentations, les pratiques effectives, révèle leurs variations à travers le temps, les sociétés et les cultures. Elle articule aussi rapport de sexe et rapport de classe. Ce ne sont pas seulement des rapports de force et de conflits (même s'ils sont essentiels) mais aussi des rapports de séduction, de consentement et d'amour, dont les formes historiques varient également.

Dans un premier temps, le « genre » a été distingué de la notion commune de sexe pour désigner les différences sociales entre hommes et femmes qui n'étaient pas directement liées à la biologie. Mais si l'invocation de la nature a servi souvent à justifier les inégalités, celle d'une construction sociale au cours de l'Histoire contribue à rendre ces inégalités plus arbitraires. Si le biologique et le social sont deux domaines distincts, alors l'idée que les inégalités de pouvoir entre hommes et femmes découlent de différences anatomiques ou de la capacité des femmes à enfanter perd de son évidence. Le genre désigne désormais la construction sociale du sexe comme champ de recherche et grille d'analyse. Féminin et masculin relèvent d'une hiérarchie dont les études de genre projettent d'explorer la construction. Toutes les sociétés ont été fondées sur cette hiérarchie et la nature a été mobilisée pour faire croire que les rôles sociaux ou les tâches dans la société étaient dévolus «naturellement » à des individus en fonction de leur sexe. Cet argument a longtemps masqué la réalité : la répartition des rôles relève d'une élaboration socio-politique. C'est ce qu'a découvert Margaret Mead dès les années 1930.

Cette ethno-anthropologue américaine $e^{3}$ a démontré qu'en Nouvelle Guinée la douceur, l'altruisme, la délicatesse, l'attention portée aux enfants, la séduction étaient des valeurs partagées par les deux sexes, alors qu'elles figuraient comme des qualités naturelles des femmes pour ses contemporains. Elle a été la première à évoquer des « rôles sexuels » dans son ouvrage Mœurs et sexualité en Océanie [2]. Elle constate qu'il n'existe pas une «nature féminine » douce et soumise, que les différences entre les sexes sont culturelles et que les rôles socio-sexuels en Occident ne sont qu'une variante parmi une infinité de possibles.

Toutefois, la distinction entre sexe biologique et genre socioculturel, bref l'invention du genre, est plutôt attribuée à des médecins psychologues

\footnotetext{
${ }_{3}^{3}$ Margaret Mead (1901-1978). Ses travaux sur la sexualité dans les cultures traditionnelles de l'0céanie et de l'Indonésie ont contribué à la révolution sexuelle des années 1960. II faut cependant signaler les controverses portant essentiellement sur un aspect de ses travaux: ceux consacrés aux adolescents de Samoa. Elle demeure une figure majeure de l'anthropologie culturelle aux États-Unis. Elle incarne les prémices d'une conception constructiviste du sexe. Toutefois, ses écrits qui présentent des limites flagrantes doivent être replacés dans leur contexte historique marqué par des représentations exotisantes de la sexualité polynésienne.
}

américains des années 1950-1960 qui ont témoigné de la divergence entre corps et identité constatée chez certains de leurs patients. Dans ses études sur la «transsexualité », Robert Stoller a séparé clairement le sexe biologique de son identification psychologique [3]. Dans ses recherches s'ancre le lexique pathologisant du «transexualisme ». Le terme transsexualité était le terme usité pour qualifier la condition des personnes qui ne s'identifiaient pas au sexe attribué à leur naissance. Ce qui était ainsi rassemblé, dans le contexte maccarthyste fortement marqué par une homophobie d'état, sous la désignation de «psychopathologies sexuelles » considérées comme troublant à la fois l'ordre des sexes et des sexualités est davantage qualifié aujourd'hui d'intersexuation. [15] $(\rightarrow)$ $(\rightarrow)$ Voir le Forum de A. Bernier et A. Leplège, $m / s$, $n^{\circ}$ 6-7, juin-juillet 2018, page 595 Cette terminologie pathologisante a été abandonnée au profit du terme d'intersexualité.

Dans les années 1960-1970, les sexologues John Money et Anke Ehrhardt insistent sur la nécessité de différencier le sexe anatomique et physiologique du genre qui renvoie à l'expérience contingente de soi comme homme ou femme. Pour eux, il faut distinguer le « rôle de genre », qui désigne les comportements «publics» d'une personne, et «l'identité de genre», qui renvoie à l'expérience privée qu'une personne a d'elle-même [4]. Chez ces psychanalystes et ces sexologues, la distinction entre sexe et genre se voulait principalement descriptive. Lorsque l'anatomie est ambiguë à la naissance, la notion de genre réfute l'évidence naturelle du sexe et indique le manque de corrélation entre les rôles sociaux et les assignations biologiques. Les études féministes vont alors se trouver en opposition avec les travaux de Money et Stoller sur un point décisif: le genre n'est pas seulement une question de savoir, c'est aussi une question de pouvoir. Les médecins, loin de remettre en cause la norme de la dualité des catégories sexuelles, poussent les individus à accéder à une supposée normalité en se conformant aux attentes sociales. Si le genre a permis de dénaturaliser le sexe, le discours «psy» des années 1950-1960, loin d'en dénoncer les conventions, participe dans un premier temps à un travail médical de normalisation, de mise en conformité des individus avec le sexe qui leur est attribué [15].

Avec la sociologue britannique Ann Oakley, l'invention du genre rencontre l'entreprise féministe de dénaturalisation du sexe. Pour elle, le sexe renvoie à la distinction biologique entre mâles et femelles, tandis que le genre renvoie à la distinction culturelle entre les rôles sociaux, les attributs et les identités psychologiques des hommes et des femmes. Le premier est pour elle 
un donné invariant tandis que le second est contingent et peut être modifié par l'action politique [5].

Avec les luttes féministes des années 1960-1970 dans les pays anglosaxons, on peut ainsi identifier une deuxième acception du concept de genre: le genre n'est pas déterminé par le sexe, il appartient à la sphère du social, du construit et du variable. Le féminisme va non seulement poursuivre la logique de dénaturalisation du genre, mais renverser la perspective pour substituer à l'entreprise de normalisation une opération résolument critique. Pour le féminisme, le genre n'est pas tant ce qu'il faut faire mais surtout ce qu'il convient de défaire : il s'agit moins de jouer le jeu des apparences que le déjouer.

Dans Penser le genre, paru en 2001, Christine Delphy, une sociologue française, interroge: "Quand on met en correspondance le genre et le sexe, on compare du social à du naturel; ou est-ce qu'on compare du social à encore du social?»([6], p. 253). La notion de genre n'échappe jamais à son ambigüité fondatrice : encore aujourd'hui, elle reste prise dans une double logique, contradictoire, entre catégorie normative et outil critique. Autrement dit, le genre est, de par son origine, une arme à double tranchant.

La philosophe américaine Judith Butler, professeure à l'université californienne de Berkeley, aujourd'hui une des principales théoriciennes du genre, souligne la dichotomie nature/culture dans Gender Trouble, paru en 1990 et traduit en français (Trouble dans le Genre) seulement en 2005 [7]. Butler y montre comment l'opération qui a arraché le genre à l'idée de nature a contribué à renforcer la division mâle/ femelle comme réalité naturelle. Poser que le genre est la part sociale du sexe risque d'alimenter l'illusion qu'une fois le genre isolé du sexe, il laisse à voir un sexe biologique vrai, purement naturel.

Thomas Laqueur renchérit dans La fabrique du sexe, parue en 1990. $\varepsilon$ montrant que la manière dont nous percevons les organes génitaux ne s'est élaborée que dans la modernité tardive, l'historien américain ébranle un présupposé majeur, celui d'une base naturelle et biologique des sexes, à laquelle le genre ne pourrait que se surajouter. Laqueur montre le passage, au XVIII ${ }^{e}$ siècle, d'un modèle unisexe hiérarchisé à un modèle moderne à deux sexes. "L'ancien modèle dans lequel hommes et femmes étaient rangés selon leur degré de perfection métaphysique, leur chaleur vitale, le long d'un axe dont le télos ${ }^{4}$ était mâle, céda la place à la fin $d u x v I I I^{e}$, à un nouveau modèle de dimorphisme radical, de divergence biologique. Une anatomie et une physiologie de l'incommensurabilité remplacèrent une métaphysique de la hiérarchie dans la représentation de la femme par rapport à l'homme » [8]. Ainsi passe$\mathrm{t}$-on d'un régime où hommes et femmes sont pensés comme de même nature à un modèle moderne où la différence des sexes serait fondée en nature.

Avec les travaux autour de Butler et Laqueur, s'observe un changement notable dans la conception de la dyade sexe/genre. Non seulement le genre n'est pas déterminé par le sexe, mais le sexe lui-même n'est plus appréhendé comme une réalité naturelle. Le genre ne désigne plus seulement les rôles individuels de sexe, mais le système qui engendre des sexes en les distinguant. Le genre ne construit pas le sexe mais

\footnotetext{
${ }^{4}$ Échelon supérieur, fin.
}

les sexes. Le genre n'est plus pensé comme un simple fait social qui pourrait s'extraire du sexe, mais comme fondamentalement un système de relations sociales produisant deux sexes posés comme antagonistes et hiérarchisés. Dès lors, le genre devient, comme pour Christine Delphy, synonyme de patriarcat et d'oppression des femmes. II renvoie à un rapport social de domination, dont il faut repérer les bénéficiaires et les opprimés. II n'est pas la part sociale de la division, il est cette division; il est un système de différenciation mais aussi de domination. Le genre est d'abord un rapport de pouvoirs (hommes/hommes, hommes/ femmes, femmes/femmes). Les femmes sont un groupe social défini à partir de leur sexe biologique et d'une construction socioculturelle du féminin, discriminante et dévalorisante. Les analyses scientifiques ne peuvent plus ignorer cette réalité, ni ignorer les questions du travestissement, de l'androgynie, des pratiques transgenres, des variations intragenres, qui reposent la question des identités. Nous retrouvons là Judith Butler, principale représentante des théories «Queer» qui insistent sur la fluidité du genre.

Le terme anglais «queer» signifie étrange. II était fréquemment utilisé comme insulte à l'égard des homosexuels ou de toute personne située hors norme de genre. Par un «retournement du stigmate», au début des années 1990, le mouvement queer fait des minorités et des identités sexuelles le lieu de contestation des normes dominantes. La critique queer promeut une politique non essentialiste d'identités par définition instables. Aujourd'hui, la critique queer a perdu de son caractère subversif et s'est normalisée dans l'institution universitaire américaine, alors qu'elle reste marginale en France. Les théories queer soulignent que l'identification du sexe d'un individu n'est pas aussi simple qu'il y paraît. Elle connaît une pluralité de critères de détermination: anatomiques (pénis/ vagin ; testicules/ovaires) ; hormonale (testostérones/ œstrogènes); génétique (chromosomes XY/XX)... Mais aucun de ces marqueurs biologiques n'assure la définition du sexe. La combinaison des critères d'expertise du sexe varie dans le temps et avec les sociétés. Ainsi, les athlètes olympiques devaient produire un certificat de féminité : jusqu'en 1968, défiler nue et montrer poitrine et vagin suffisaient. Face à certains cas ambigus, des critères plus «scientifiques » (génétiques) ont été exigés, mais il s'est trouvé des cas difficiles comme Maria Patino, coureuse olympique disqualifiée sur la base du test $A D N$ mais qui vécut toute sa vie comme femme. Depuis 2000, le Comité olympique international (COI) n'impose plus d'examen systématique aux femmes, mais se réserve le droit d'exiger un test en cas de 
« doute visuel ». C'est ainsi qu'après avoir remporté la médaille d'or aux championnats du monde de Berlin en 2009, la coureuse sud-africaine Caster Semenya, aux traits considérés comme masculins, a subi dix mois d'examens humiliants pour déterminer son identité sexuelle. Les examens ont conclu qu'elle était bien une femme, mais avec une hyperandrogénie. L'excès de sécrétion de testostérone favoriserait développement musculaire, endurance et vitesse. Tirant les leçons de l'épisode, le COI a élaboré en 2012 un nouveau règlement relatif à l'hyperandrogénie féminine, permettant de vérifier le taux de testostérone d'une athlète. Le $1^{\text {er }}$ mai 2019 , le tribunal arbitral du sport a débouté Caster Semenya qui souhaitait invalider le règlement de la Fédération internationale d'athlétisme (IAAF) imposant aux femmes hyperandrogènes de prendre des médicaments afin de faire baisser leur taux de testostérone pour participer aux épreuves internationales sur les distances allant du $400 \mathrm{~m}$ au $1500 \mathrm{~m}$. Selon cette décision reconnue comme discriminatoire par le tribunal (!), Caster Semenya devra donc se plier au nouveau règlement et suivre un traitement dont elle ne veut pas, si elle souhaite poursuivre la compétition.

De l'avis de nombreux experts, aucun critère unique ne permet d'établir la différence entre les hommes et les femmes, et le critère hormonal n'est pas plus pertinent que les précédents. D'ailleurs, le CIO se garde bien de chiffrer officiellement le taux féminin «normal», attendant d'avoir un cas pour statuer. Selon I'endocrinologue britannique Peter Sonksen, sur 650 olympiens, $5 \%$ des femmes avaient un niveau «masculin » de testostérone et $6 \%$ des hommes un niveau «féminin ». «La différence de taux est en effet parfois plus importante entre deux hommes qu'entre un homme et une femme », rappelle Anaïs Bohuon, chercheuse à Paris-Sud, auteur du livre intitulé Test de féminité dans les compétitions sportives: une histoire classée $X$ ? [9]. Surtout, rien ne prouve que cette hormone soit déterminante dans la performance d'un sportif. «Mais même si l'hyperandrogénie représentait un atout, pourquoi faudrait-il le sanctionner? » poursuit Anaïs Bohuon. "Les athlètes de haut niveau ont souvent, par définition, des caractéristiques naturelles hors norme ». Retenir un critère particulier n'a aucun sens. «On ne pénalise pas Michael Phelps parce qu'il a de très grands pieds, ou Jeannie Longo parce qu'elle a un cœur qui bat très lentement». Cibler la testostérone chez les femmes est d'autant plus injuste que "certains hommes ont des taux plus élevés que la moyenne, et ils ne sont jamais inquiétés », ajoute Anaïs Bohuon. En fait, la raison d'être de ces tests ne serait pas vraiment d'éviter la tricherie. "Jamais dans l'histoire des compétitions sportives un homme n'a tenté de se faire passer pour une femme, affirme la chercheuse. Cet acharnement sur le corps féminin traduit avant tout une volonté de conserver l'infériorité "naturelle" de la femme par rapport à l'homme ».

Les nombreux travaux sur l'intersexualité (synonyme d'hermaphrodisme ou la situation d'êtres humains possédant des caractéristiques de sexe indéterminées ou ne permettant pas une identification classique en mâles ou femelles) remettent en cause la distinction radicale des deux sexes. Le sexe représente un ensemble de données et il n'y a pas d'élément unique permettant de considérer qu'on est soit mâle soit femelle. La médecine va devoir définir le «vrai » sexe de 1,7\% des enfants naissant avec des caractéristiques indéterminées. Le mouvement transgenre (personne qui revendique une identité trans en tant que telle et non l'appartenance à une catégorie de sexe homogène) remet en cause la bicatégorisation du genre et la prétention à faire du sexe un indice pertinent de division du monde social. Kate Bornstein dit dans Gender Outlaw : on Men, Women, and the Rest of Us ([10], p. 337) : « je n'ai jamais haï mon pénis, j'ai haï le fait qu'il faisait de moi un homme ».

Le genre, au départ, c'était la construction sociale du sexe biologique. La différence des sexes n'est pas une donnée de nature immuable; elle n'existe que dans I'Histoire. Ce que c'est qu'être un homme ou une femme ne peut être abstrait du contexte social. Le sexe est indissociable des normes sexuelles qui, par définition, ne sont pas naturelles. Si ces normes sont susceptibles de changer, cela veut dire qu'elles sont un enjeu politique.

La société continue en effet à reproduire une norme masculine dominante. Les livres pour enfants, les catalogues de jouets, les jeux vidéos continuent à véhiculer des stéréotypes souvent décalés par rapport à la réalité d'aujourd'hui : le petit garçon est très actif, courant partout, conquérant l'espace, la petite fille, plus volontiers à la fenêtre, regarde au dehors, figée, passive. II y a aussi le code des couleurs : rose et bleu... Françoise Héritier a montré comment, à partir d'une observation de différences, s'est élaboré un système hiérarchique au détriment du féminin, dont nombre de traces sont encore perceptibles [11]. Pourtant le système craque de partout, ne correspond plus aux mutations en cours, aux aspirations individuelles et collectives à la liberté, à l'épanouissement et la singularité de chacun, chacune. Le genre enrichit la panoplie des outils à la disposition des chercheurs et est en passe de devenir un langage privilégié de la modernité démocratique. Éric Fassin parle de «démocratie sexuelle », soit de la dénaturalisation en marche de l'ordre des sexes et des sexualités, au nom des principes politiques de liberté et d'égalité [12]. Les historiens du genre proposent un découpage alternatif de la matière historique: tout phénomène ou événement peut également être observé du point de vue des femmes et prend alors d'autres dimensions et d'autres significations. L'histoire du genre remet en cause la périodisation qui résulte d'une hiérarchisation des événements. Joan Kelly conteste, par exemple, la notion et le terme de Renaissance: les femmes ne connaissent pas de Renaissance dans la période ainsi nommée (pas plus que dans la Nahda

\footnotetext{
${ }^{5}$ Mouvement de renouveau littéraire et politique au Moyen-Orient.
} 
arabe du XIX $x^{e}$ siècle) [13]. Le genre devient dès lors un élément structurant des disciplines. Le triptyque race/classe/genre renouvelle les réflexions sur les inégalités socio-économiques.

Les rapports de pouvoir sont en perpétuelle capacité d'inversion, de réajustement et sans cesse reconfigurés dans des contextes historiques multiples. Mais les recherches sur les sociétés humaines montrent que cette asymétrie se construit quasiment toujours au profit des hommes, avec une valorisation systématique du masculin, exprimée par exemple par le concept de «patriarcat». Le genre renvoie à un rapport de domination des hommes sur les femmes et à une sanction des «déviances 》 (par exemple des «hommes efféminés », des lesbiennes «butch»[ « femmes masculines»], des personnes «trans », etc.).

Les rapports de genre sont toujours imbriqués dans des rapports de pouvoir. Cette dimension est appelée couramment «intersectionnalité », depuis l'article fondateur de la juriste américaine Kimberlé Crenshaw, Mapping the Margins: Intersectionality, Identity Politics, and Violence against Women of Color [14]. Croisant le genre avec d'autres rapports de pouvoir (classe, race, etc.), la perspective intersectionnelle rend l'analyse du genre encore plus complexe en s'adressant aux expériences sociales singulières. Elle s'est imposée sous l'effet de luttes au sein du mouvement féministe: dans les années 1970, aux États-Unis, les militantes afroaméricaines du Black Feminism ont dénoncé le caractère exclusif de théories féministes forgées à l'aune des expériences des femmes blanches hétérosexuelles des classes moyennes et supérieures. Elles ont mis en exergue les rapports de pouvoir entre femmes, sous l'effet combiné du racisme et de la domination de classe. Ces analyses critiques ont été complétées par les Subaltern Studies à partir des années 1980 et par les recherches poststructuralistes et queer des années 1990.

Le sexe et le genre ne sont pas des variables séparées: il y a interaction entre la biologie et l'environnement social dès la naissance, voire avant. II est important que les médecins considèrent désormais les différences entre hommes et femmes non pas comme une simple dichotomie mâle et femelle, mais comme le produit d'une intrication entre sexe et genre. Penser la médecine à la lumière du sexe et du genre apporte une vision plus précise des inégalités de santé et de leur origine.

\section{L'interaction entre sexe et genre en santé}

L'état de santé d'une personne peut être influencé par son activité professionnelle, son niveau d'études et son hygiène de vie. Ces déterminants diffèrent souvent chez les hommes et chez les femmes, selon le contexte social et culturel. Par exemple, les hommes, majoritairement, tardent plus à consulter que les femmes. La relation médecin-patient peut être également influencée (bien souvent inconsciemment) par les représentations sociales liées au genre. Les recherches en sciences sociales montrent combien les codes sociaux de la féminité (fragilité, sensibilité, expression verbale) et de la masculinité (virilité, résistance au mal, prise de risque) jouent un rôle dans l'expression des symptômes, le rapport au corps et le recours aux soins. Ces stéréotypes influencent la façon dont les professionnel.les de santé dépistent et prennent en charge certaines maladies. L'articulation sexe/genre permet de s'interroger sur les différences en matière de santé, afin d'apporter une réponse adaptée. Elle fait l'objet d'une réflexion éthique qui vise à favoriser de nouvelles pratiques dans la recherche et la clinique.

\section{Quelques illustrations}

- L'infarctus du myocarde est un exemple typique d'interaction entre sexe et genre. Le risque d'infarctus a longtemps été associé à l'image d'un homme d'âge mûr stressé au travail. De ce fait, la maladie est sousdiagnostiquée chez les femmes. Une patiente qui se plaint d'oppression dans la poitrine se verra prescrire des anxiolytiques alors qu'un homme sera orienté vers un cardiologue. Les symptômes d'infarctus peuvent par ailleurs se manifester de façon «atypique » chez les femmes. Le risque de retard au diagnostic et à la prise en charge est attesté : la majorité des décès liés à l'infarctus concernent aujourd'hui les femmes.

- À l'inverse, l'ostéoporose est sous-diagnostiquée chez les hommes, car elle est considérée comme une maladie des femmes ménopausées. Or un tiers des fractures du col du fémur chez les hommes sont dues à l'ostéoporose. En conséquence, le risque de deuxième fracture est aussi élevé chez eux que chez les femmes.

- La dépression est une maladie souvent considérée comme essentiellement féminine, mais elle apparaît fréquemment chez les hommes lorsque l'on intègre comme critères diagnostiques des symptômes tels que l'agressivité et l'augmentation des comportements à risque (consommation d'alcool, de drogues, conduite automobile dangereuse).

Les exemples de l'influence du sexe et du genre sur la santé concernent quasiment tous les champs de la médecine: cancer, diabète, obésité, douleur, asthme, arthrose, addiction, vieillissement, etc.

L'étude des liens entre sexe, genre et santé représente donc une approche transversale intégrant la globalité de l'être humain, pour comprendre ou résoudre les problèmes de santé. Elle implique une évolution méthodologique. Dans le cadre des recherches précliniques (in vitro ou chez l'animal), la question du sexe des cellules et de celui des animaux dans les travaux de recherche se pose. Sur le plan cellulaire, et même en dehors des cellules sexuelles (gonades, gamètes), certains mécanismes diffèrent en fonction du sexe dans divers tissus. Si ces données au niveau microscopique sont documentées, leurs conséquences au niveau de l'organisme entier sont mal connues, en particulier dans l'espèce humaine. Ouvrir la recherche médicale à la question du 
sexe et du genre amène donc à questionner la validité des modèles classiques des protocoles de recherche et à en construire de nouveaux. Cette innovation en médecine devrait intervenir pour le plus grand bénéfice des femmes et des hommes.

\section{Conclusion}

Le genre n'est pas un nouveau terme pour parler d'hommes, de femmes, de féminin, de masculin ou de différence des sexes. Le genre est une manière nouvelle de les percevoir en adoptant une posture déconstructiviste (qui s'oppose à l'essentialisme), qui révèle entre autres des rapports de pouvoir entre le féminin et le masculin, euxmêmes imbriqués dans d'autres rapports de pouvoir.

Il ne s'agit pas de constituer un nouveau champ clos du savoir qui serait les études de genre, mais de contribuer à un réexamen des notions clés des disciplines scientifiques. Si les démarches et les méthodes ne diffèrent pas radicalement, la question posée, elle, est radicalement différente. Quel est son pouvoir de transformation du champ du savoir? Comment peut-elle changer concrètement la pratique? L'expérience vaut d'être tentée. $\diamond$

Sex, gender, ethics

\section{LIENS D’INTÉRÊT}

L'auteure déclare n'avoir aucun lien d'intérêt concernant les données publiées dans cet article.

\section{RÉFÉRENCES}

1. Testard-Vaillant P. Les sciences du vivant ont besoin du genre. CNRS Le Journal, mars 2014.

2. Mead M. Mœurs et sexualité en Océanie. Paris : Plon ; 1969 : 534 p.

3. Stoller R. Sex and gender. On the development of masculinity and feminity. New York : Karnac Books; 1968 : 384 p.

4. Money J, Ehrhart A. Man and woman, boy and girl. Baltimore : The Johns Hopkins University Press ; 1972 : 312 p.

5. Oakley A. Sex, gender and society. Londres : Temple Smith ; 1972 : 184 p.

6. Delphy C. Penser le genre. Paris : Syllepse ; 2001 : 366 p.

7. Butler J. Gender trouble. New York-Londres : Routledge ; $1990: 284$ p.

8. Laqueur T. La fabrique du sexe. Paris : Gallimard ; $1992: 348$ p.

9. Bohuon A. Test de féminité dans les compétitions sportives : une histoire classée X? Paris : Éditions iXe ; 2012 : 192 p.

10. Bornstein K. Gender outlaw : on men, women, and the rest of us. New YorkLondres : Routledge ; 1994 : 320 p.

11. Héritier F. Masculin/féminin. La pensée de la différence. Paris : Odile Jacob, $1996: 332 \mathrm{p}$.

12. Fassin $\varepsilon$. La démocratie sexuelle et le conflit des civilisations. Multitudes $2006 ; 3: 123-31$.

13. Kelly J. Did women have a Renaissance? In : Bridenthal R, Koonz C, eds. Becoming visible, women in European history. Boston : Houghton Mifflin ; 1977 : 21-47.

14. Crenshaw KW. Mapping the margins: intersectionality, identity politics, and violence against women of color. Stanford Law Review 1991 ; 43 : 1241-99.

15. Bernier A, Leplège $A$. Les traitements hormonaux des mineurs transgenres, ou les obstacles de l'éthique médicale aujourd'hui. Med Sci (Paris) 2018 ; $34: 595-8$.

16. Vidal C., Salle M. Femmes et santé, encore une affaire d'hommes? Paris : Berlin, $2017: 80$ p.

\section{TIRÉS À PART}

K. Lambert

\section{WwW.myobase.org}

Catalogue en ligne disponible gratuitement sur Internet publié par l'AFM-Téléthon. Retrouvez facilement toutes les références bibliographiques sur les maladies neuromusculaires, les situations de handicap qu'elles génèrent et leurs aspects psychologiques.

Myobase donne un accès libre à $75 \%$ du fonds documentaire collecté depuis 1990 , représentant plus de 40000 références spécifiques du domaine des maladies neuromusculaires.

> articles de la littérature biomédicale

et psycho-sociale

$>$ livres, thèses

$>$ guides d'associations et rapports institutionnels d'agences internationales

$>$ brèves en français, synthèses des articles médico-scientifiques internationaux les plus pertinents

\section{$>$ publications}

AFM-Téléthon destinées aux professionnels de santé ou aux personnes atteintes de maladie neuromusculaire et à leur entourage
UN OUTIL ERGONOMIQUE, UNE INTERFACE BILINGUE

- Laissez-vous guider par les tutoriels

- Lancez une recherche et affinez votre sélection grâce aux filtres

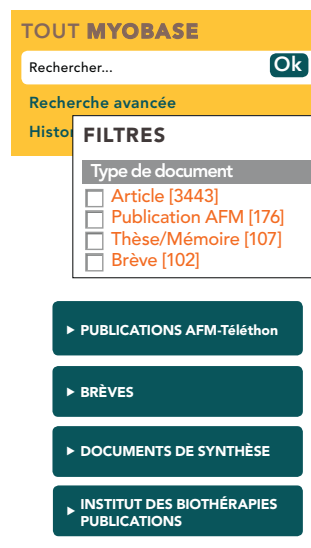

- Partagez les résultats de votre recherche
UN ACCÈS facile et simple

Rechercher avec des opérateurs :

- guillemets pour une expression "maladie de pompe"

- + pour signifier ET, et retrouver tous les documents contenant les deux mots "fauteuil +électrique"

- pour signifier NON et enlever le mot de la recherche

"autonomie -établissement"

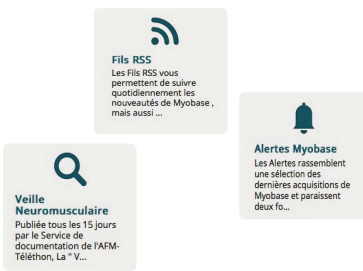

- Cliquez sur l'onglet

thématique qui vous convient

(haut de la page d'accueil)

- Créez vos alertes personnalisées en ouvrant un compte personnel

- Téléchargez la Veille Neuromusculaire

- Abonnez-vous aux flux RSS 